\title{
Novel Information on Anatomic Factors Causing Grasp Reflex in Frontal Lobe Infarction: A Case Report
}

\author{
Ikjun Choi, MD, Kwang-Ik Jung, MD, Woo-Kyoung Yoo, MD, Soyeon Jang, MD, Suk Hoon Ohn, MD \\ Department of Physical Medicine and Rehabilitation, Hallym University Sacred Heart Hospital, \\ Hallym University College of Medicine, Anyang, Korea
}

We report a patient with a severe limitation of function in the right hand resulting from grasp reflex following a stroke affecting the left anterior cerebral artery region. We describe, using diffusion tensor tractography (DTT), a disconnection between the bilateral frontal lobes via the corpus callosum. The patient could not control his right hand at all, even though his bilateral corticospinal tracts were intact. We noted that over the infarcted lesion on DTT, the white matter was invisible from the corpus callosum to the prefrontal cortex. These findings reflected a unique pattern of white-matter disconnection between the ipsilateral medial frontal lobe and ipsilateral and contralateral frontal cortex causing hand function deterioration in the form of severe grasp reflex.

Keywords Grasp reflex, Frontal lobe, White matter

\section{INTRODUCTION}

Rehabilitation physicians' treatment planning for stroke patients with unusual symptoms needs to take due account of the alteration of the brain's neuronal integrity. The frontal lobe, with its intricate connections with other brain systems, plays a number of complex roles that are critical to goal-directed behavior; damage incurred to the frontal lobe accordingly leads to loss of various mo-

Received June 18, 2014; Accepted August 1, 2014

Corresponding author: Suk Hoon Ohn

Department of Physical Medicine and Rehabilitation, Hallym University Sacred Heart Hospital, Hallym University College of Medicine, 22 Gwanpyeong-ro 170beon-gil, Dongan-gu, Anyang 431-796, Korea Tel: +82-31-380-3860, Fax: +82-31-380-3864, E-mail: ohnsh@hallym.ac.kr

(c) This is an open-access article distributed under the terms of the Creative Commons Attribution Non-Commercial License (http://creativecommons. org/licenses/by-nc/3.0) which permits unrestricted noncommercial use, distribution, and reproduction in any medium, provided the original work is properly cited.

Copyright (C) 2015 by Korean Academy of Rehabilitation Medicine tor functions. What is especially helpful for understanding such patients' symptoms is neural network analysis. Diffusion tensor tractography (DTT), for instance, is a recently developed brain-mapping technique that can visualize the anatomical connectivity of subcortical neural tracts. Our earlier study employed DTT for visualization of the corticothalamic tract, which enabled us to understand the mechanism of response to repetitive transcranial magnetic stimulation (rTMS) in post-stroke centralpain patients [1]

Grasp reflex is a rare phenomenon whereby tactile pressure on the palm triggers the contraction of the flexor and adductor muscles of the hand. Rarely, stretching of the contracted muscles or the attempt to withdraw the stimulating object exacerbates the contraction, thus resulting in further strengthening of the grasp [2]. Grasp reflex has been reported in patients with damage to the medial frontal lobe and anterior cingulated gyrus, both of which structures, significantly, are constituted mainly 
of bi-hemisphere connection fibers [3]. Therefore, grasp reflex is best conceived as an aspect of inter-hemispheric or intra-hemispheric fiber-disconnection syndrome.

In these pages we present the case of a right-hemiparetic patient suffering grasp reflex after left frontal lobe infarction. For the first time with a grasp reflex patient, we utilized DTT to describe the status of the inter-hemispheric white-matter connection.

\section{CASE REPORT}

A 40-year-old man who developed a cerebral infarction on the left medial frontal lobe including the cingulate gyrus, supplementary motor cortex and substantia nigra (Fig. 1A) was transferred to our department on the 10th post-stroke day. The patient presented with right hemiparesis, anomic aphasia, depressive mood, mild cognitive impairment and exacerbated grasp reflex accompanied by difficulty of release in the right hand. He had no history of neurological or orthopedic disease. Muscle strength, rated using the manual muscle test, was grade 3/5 in the right upper and lower limbs. Synergic muscular contraction did not occur during arm motion. The patient's grasp reflex manifested as follows: upon contact of the palm with any kind or size of object, the object was grasped involuntarily and very tightly, and could not be released independently. Tactile pressure on the palm triggered forceful contraction of the flexor muscles of the wrist and fingers. Stretching of the contracted muscles only exacerbated their contraction. These symptoms,
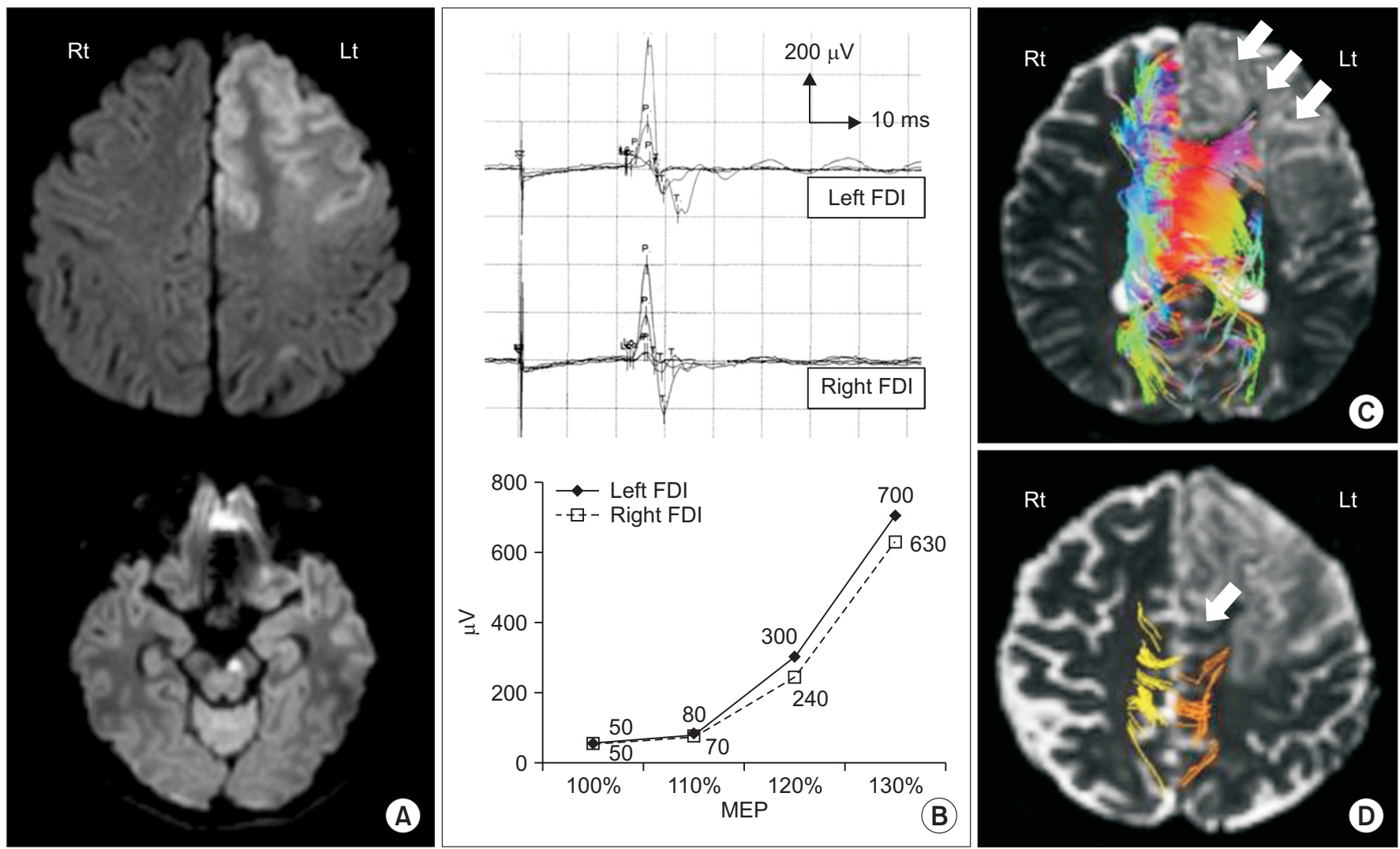

Fig. 1. (A) Diffusion-weighted image of patient. Magnetic resonance imaging shows cerebral infarction in left anterior cerebral artery region including left medial frontal lobe, prefrontal cortex and substantia nigra. (B) Motor-evoked potential (MEP) amplitudes recorded in right and left first dorsal interossei (FDI) muscles. The resting MEP threshold, latency (upper panel) and increase (lower panel) were similar between the muscles. (C) Color diffusion tensor tractography (DTT) of transcallosal fibers. These fibers did not appear in the corpus callosum or left prefrontal cortex in the infarcted area (white arrows). In the color DTT, each color indicates the white-matter direction: red, anteriorposterior; blue, cephalic-caudal; green, right-left. (D) DTT of corticospinal tracts (CST). The left CST was somewhat more affected than the right (white arrow). 
causing severe restriction of right-hand function, were worsened by emotion and stress.

Motor-evoked potentials (MEPs) were obtained, according to the same protocol used in our previous study [1]. The resting MEP threshold and latency were $44 \% / 23.4$ $\mathrm{ms}$ and $50 \% / 23.0 \mathrm{~ms}$ in the left and right first dorsal interossei (FDI) muscles, respectively. The MEP amplitudes, measured at $100 \%, 110 \%, 120 \%$ and $130 \%$ of the resting MEP threshold, are plotted in Fig. 1B.

Diffusion tensor imaging (DTI) data also were obtained, again according to the same protocol as used in our previous study [1]. The diffusion tensor was calculated, and 3-dimensional tractography of the transcallosal fibers and corticospinal tracts (CST) was performed using MedINRIA software (INRIA Sophia Antipolis, France). For the tractography of the transcallosal fibers, two 2-dimensional regions of interest (ROIs) were manually placed on the margins of the right and left corpus callosum according to Mori's method [4]. For the CST, three ROIs defined the motor cortex, anterior pons and lower medulla. The threshold of tracking termination was set at a fractional anisotropy (FA) value of 2.0, and the angle between two contiguous eigenvectors was set as $30^{\circ}$. The transcallosal fibers could not be visualized in the affected hemisphere (Fig. 1C). The CSTs could be visualized in both the unaffected and affected hemispheres (Fig. 1D). The FA and apparent diffusion coefficient were $0.417 / 0.360$ and $2.122 / 2.331$, respectively, in both the unaffected and affected hemispheres.

\section{DISCUSSION}

Analysis of the DTT and MEP results provided novel information on the anatomic factors that contribute to the development of grasp reflex in stroke patient. In this patient, DTT revealed damage to the transcallosal fibers between the corpus callosum and left prefrontal cortex. Both DTT and MEP showed the left CST to be somewhat more affected than the right. All of the diffusion indices were similar in the affected and unaffected hemispheres. Among the MEP parameters, latency is regarded as the most reliable for evaluation of pyramidal tract function, while amplitude and threshold are regarded as better markers of the degree of cortical and pyramidal tract activation [5]. The latency, amplitude and threshold of the MEPs evoked by various stimulations of differing inten- sity were similar in the affected and unaffected hands. Therefore, the patient's grasp reflex was considered to have arisen from a non-CST-related motor network problem.

Either inter-hemispheric or intra-hemispheric whitematter disconnection can cause grasp reflex. Inter-hemispheric disconnection occurs by lesions involving the medial frontal lobe (including the cingulate gyrus) or the supplementary motor area [3]. These areas, connected by transcallosal fibers, are related to planning and inhibitory control for coordinated motor tasks; a lesion of any of these areas, therefore, might affect deftness of movement $[5,6]$. Successful motor tasks require an intact motor and praxis system, in the parietal lobe of which, motor planning information is stored preparatory to translation to the prefrontal lobe. During translation between the ipsilateral parietal and frontal lobes, the executive motor controls are detailed by positive and negative modulation from the contralateral frontal lobe via transcallosal fibers. This consolidated motor planning is then transferred to the ipsilateral primary motor cortex, which activates the CST. Thus, transcallosal fiber lesions have negative influences on the motor and praxis system, and in some cases, induce grasp reflex [7].

In our patient, grasp reflex might have been aggravated by the intra-hemispheric disconnection of the indirect dopamine pathway, which also is important for motor control. The indirect dopamine pathway forms, with the direct dopamine pathway, the cerebral dopamine networks. The direct pathway projects to the internal segment of the globus pallidus and substance nigra, and plays a role in initiating and maintaining movement. The indirect pathway, meanwhile, which runs through the external segment of the globus pallidus in the medial frontal lobe, stabilizes movement [8]. As a result, damage to the medial frontal lobe compromises the excitatory and inhibitory control of the dopamine pathways and effects, thereby, the grasp reflex phenomenon.

In this present investigation, DTT was used to visualize the neural bases of a disconnection syndrome, similarly to previous studies on post-stroke central pain, cognitive impairment and neglect $[1,9]$. rTMS, specifically by long-term potentiation and dopamine release $[1,10]$, was proved to modulate the disconnection status in patients with post-stroke central pain. rTMS therefore might be utilized hereafter in the treatment of grasp reflex. 


\section{CONFLICT OF INTEREST}

No potential conflict of interest relevant to this article was reported.

\section{REFERENCES}

1. Ohn SH, Chang WH, Park CH, Kim ST, Lee JI, PascualLeone A, et al. Neural correlates of the antinociceptive effects of repetitive transcranial magnetic stimulation on central pain after stroke. Neurorehabil Neural Repair 2012;26:344-52.

2. Seyffarth H, Denny-Brown D. The grasp reflex and the instinctive grasp reaction. Brain 1948;71:109-83.

3. Hashimoto R, Tanaka Y. Contribution of the supplementary motor area and anterior cingulate gyrus to pathological grasping phenomena. Eur Neurol 1998;40:151-8.

4. Mori S, Kaufmann WE, Davatzikos C, Stieltjes B, Amodei L, Fredericksen $\mathrm{K}$, et al. Imaging cortical association tracts in the human brain using diffusion-tensor- based axonal tracking. Magn Reson Med 2002;47:21523.

5. Wassermann EM, Epstein CM, Ziemann U. The Oxford handbook of transcranial stimulation. Oxford: Oxford University Press; 2008.

6. Sumner P, Nachev P, Morris P, Peters AM, Jackson SR, Kennard C, et al. Human medial frontal cortex mediates unconscious inhibition of voluntary action. Neuron 2007;54:697-711.

7. Heilman KM, Watson RT. The disconnection apraxias. Cortex 2008;44:975-82.

8. Alexander GE, Crutcher MD. Functional architecture of basal ganglia circuits: neural substrates of parallel processing. Trends Neurosci 1990;13:266-71.

9. Molko N, Cohen L, Mangin JF, Chochon F, Lehericy S, Le Bihan D, et al. Visualizing the neural bases of a disconnection syndrome with diffusion tensor imaging. J Cogn Neurosci 2002;14:629-36.

10. Sanes JN, Donoghue JP. Plasticity and primary motor cortex. Annu Rev Neurosci 2000;23:393-415. 